\title{
GIANT RETINAL TEARS AFTER PARS PLANA VITRECTOMY
}

\author{
AHMED M. ABU EL-ASRAR \\ Riyadh, Saudi Arabia
}

\section{SUMMARY}

Vitreous surgery is used to treat complicated vitreoretinal pathology. Retinal tears are a serious complication of vitreous surgery. In this report, the development of giant retinal tears in four eyes after pars plana vitrectomy for posterior segment pathology is described. The pathogenesis and implications of this serious and infrequent complication are discussed.

Giant retinal tear was originally defined as a retinal tear greater than 3 clock-hours in circumferential extent. ${ }^{1}$ Subsequently, $\mathrm{Scott}^{2}$ pointed out that the essential feature of a giant tear is independent mobility of the posterior flap. Most such tears are idiopathic and occur as a result of separation of the vitreous as far forwards as the ora serrata. The giant oral tear results from a separation of the pars plana which takes place at the time of vitreous detachment. The vitreous remains attached to the detached pars plana which forms the anterior flap of the tear. The posterior flap forms secondarily, is free of vitreal attachment, and acquires independent mobility. ${ }^{2}$ Freeman $^{3}$ suggested that spontaneous giant tears occur as a result of syneresis and liquefaction of the vitreous gel and condensation of the remaining anterior vitreous with membrane formation along its posterior aspect. Contraction of this membrane results in the formation of a giant retinal tear along the posterior border of the vitreous base.

The second most common cause of giant retinal tears is blunt trauma, and in these cases there is usually avulsion of the vitreous base throughout all or part of the length of the tear. ${ }^{1}$ The proposed mechanisms for the formation of blunt traumatic giant retinal tears involve a transient reduction in the axial length during impact. Scott ${ }^{2}$ had suggested that retrodisplacement of the cornea and aqueous drives the lens-iris diaphragm back against the anterior

Correspondence to: Dr Ahmed M. Abu El-Asrar, Department of Ophthalmology, College of Medicine, King Saud University, PO Box 55307, Riyadh 11534, Saudi Arabia. Fax: (966)-1-4775791. vitreous face away from its adherence to the vitreous base. Because the extensibility of the sclera is greater than that of the vitreous, the sclera expands and oscillates with a greater amplitude. As a result the vitreous base and attached ciliary epithelium pull away from the pigment epithelium of the pars plana ciliaris. ${ }^{4}$ There is evidence that a high-speed deformation of the cornea and sclera may be more effective in producing damage to the fundus periphery than the amount of kinetic energy applied. ${ }^{4}$ Giant retinal tears occur frequently in patients with Wagner-Stickler syndrome. ${ }^{5}$ In addition, McLeod reported the development of giant retinal tears in four eyes after central pars plana vitrectomy. ${ }^{6}$

In this report, the development of giant retinal tears in four eyes that had previously undergone pars plana vitrectomy for posterior segment pathology is described.

\section{Case 1}

\section{CASE REPORTS}

A 37-year-old man was referred 15 days after sustaining penetrating injury to his right eye while hammering on a piece of metal. On examination, the patient was found to have a visual acuity of hand motion in the right eye, which showed self-sealed corneal laceration and total cataract. A computed tomographic scan revealed an intravitreal metallic foreign body. The patient underwent pars plana lensectomy and vitrectomy, and foreign body removal using an intraocular foreign body forceps. After completing the procedure $360^{\circ}$ indirect ophthalmoscopy with scleral indentation revealed no untoward retinal abnormality, whether pre-existing or surgically induced. Vision improved to $6 / 24$ with an aphakic correction.

Sixteen days post-operatively, follow-up funduscopy revealed complete separation of the residual vitreous skirt as far forwards as the ora serrata, together with a $120^{\circ}$ nasal giant retinal tear $(2.30$ o'clock clockwise to 6.30 o'clock). The residual 
mobile peripheral vitreous gel was attached to the raised anterior flap of the tear which was formed by the detached non-pigmented pars plana epithelium. The posterior flap of the tear was mobile with a shallow retinal detachment that extended about $1.5 \mathrm{~mm}$ beyond the edge of the tear. The patient was immediately treated with two rows of confluent laser photocoagulation burns which were applied to the peripheral retina at the border between attached and detached retina and were extended to $360^{\circ}$. When the patient was last seen 2 years later, examination showed a flat attached retina posterior to the photocoagulation scars.

\section{Case 2}

A 30-year-old man was referred for management of a retinal detachment in his myopic right eye $(-0.6 \mathrm{D})$. Examination revealed a visual acuity of counting fingers at $1 \mathrm{~m}$ in the right eye. Funduscopy showed total bullous rhegmatogenous retinal detachment secondary to multiple horseshoe tears. The right eye underwent encircling band application, pars plana vitrectomy, heavy liquid injection, cryopexy applied to retinal tears, and fluid/20\% $\mathrm{SF}_{6}$ gas exchange. Post-operatively, the retina became reattached and vision improved to 6/24.

Six months post-operatively, routine funduscopy revealed an inferonasal shallow retinal detachment secondary to a $100^{\circ}$ giant retinal break at the ora serrata (2.30 o'clock clockwise to 6.00 o'clock). Peripheral residual mobile vitreous was completely separated from the retina and was attached to the anterior flap of the giant break. The posterior flap of the tear was partially inverted. A further retinal reattachment procedure was therefore performed. The detached vitreous cortex was removed, and the posterior flap of the tear was manipulated into place using heavy liquid injection, followed by cryopexy application to the repositioned edge of the posterior flap and through $360^{\circ}$. Fluid/silicone oil exchange was then performed. Two months after the second procedure, the silicone oil was removed. Ten months later, right vision was $6 / 24$. There was mild posterior subcapsular cataract, and the retina was entirely attached.

\section{Case 3}

A 24-year-old man was injured in the left eye by a wooden stick. On presentation to the emergency room 5 hours later, he had a corneal laceration with iris incarceration, shallow anterior chamber and total cataract. A few hours later, the patient underwent primary wound repair combined with sampling from the injury site for microbiological analysis. Marked anterior chamber reaction was noted 24 hours after primary repair. Ultrasonography showed dense vitreous opacities with multiple strands and mem- branes, diffuse thickening of the retina-choroid layer, and the retina was attached, confirming the diagnosis of endophthalmitis. The patient underwent pars plana lensectomy and diagnostic and therapeutic pars plana vitrectomy, followed by intravitreal injection of amikacin and vancomycin, and subconjunctival injection of vancomycin, amikacin and triamcinolone. No oral or peripheral retinal abnormality was detected at the conclusion of the procedure by $360^{\circ}$ indirect ophthalmoscopy with scleral indentation. Microbial cultures showed haemolytic streptococci. One month later, the inflammation gradually subsided and the patient's vision in this eye improved to $6 / 24$.

Two months post-operatively routine follow-up retinal examination showed a completely detached mobile residual peripheral vitreous cortex and an inferotemporal $100^{\circ}$ giant retinal tear at the ora serrata (2.30 o'clock clockwise to 6.00 o' clock) with a raised anterior flap from which the vitreous remnant was suspended. There was slight lifting of the posterior flap, but the retina was otherwise flat. Prophylactic $360^{\circ}$ post-oral laser photocoagulation was performed. Five months later, the retina was entirely attached posterior to the photocoagulation scars.

\section{Case 4}

A 23-year-old man was referred 10 days after undergoing pars plana vitrectomy and removal of an intraocular foreign body in the left eye. Eight days after the procedure the patient was noted to develop retinal detachment due to giant retinal tear. At presentation the patient's visual acuity was $6 / 12$ in the left eye. Funduscopy examination revealed detached residual peripheral vitreous cortex which was mobile and temporal retinal detachment not including the macula due to a temporal $120^{\circ}$ giant retinal tear at the ora serrata $(1.00$ o'clock clockwise to 5.00 o'clock). The anterior flap was elevated by the attached vitreous gel remnant. The posterior flap of the tear was inverted. The patient underwent encircling band application, and removal of the detached vitreous cortex. The posterior flap of the break was manipulated into place using heavy liquid injection. Cryopexy was applied to the repositioned edge of the posterior flap and through $360^{\circ}$, and fluid silicone oil exchange was performed. Two months after the second procedure, silicone oil was removed with peeling of an epimacular membrane. Four months later, the retina was entirely attached and the patient's vision in that eye was $6 / 24$.

\section{COMMENT}

Retinal tears are a serious and relatively frequent complication of vitreous surgery. Peripheral retinal tears can occur along the posterior margin of the 
vitreous base and are most common in the meridians of pars plana sclerotomies, occurring in $2 \%^{7}$ to $8 \%^{8}$ of cases. These tears are probably due to traction on the adjacent vitreous base from the vitrectomy instruments. An oral giant retinal tear occurring during vitrectomy for the repair of the late complications of diabetic retinopathy was noted (John Scott, personal communication). This peroperative mechanism of giant retinal tear formation was excluded in patients 1,2 and 3 by the routine practice of $360^{\circ}$ indirect ophthalmoscopy with scleral indentation after completing the procedure. Patient 4 was managed at our unit after referral, and it was not clear whether the giant retinal tear developed at the time of pars plana vitrectomy or subsequently.

The giant retinal tears in patients 1,2 and 3 were probably related to detachment of the residual peripheral vitreous after surgery, combined with pre-existing or surgically induced oral pathology. Alternatively, post-operative fibrovascular ingrowth from the incision may be implicated. Contraction of this fibrovascular tissue can pull away the pars plana from the ora serrata leaving an unsupported retina behind the ora forming a posterior flap. Delayed retinal breaks were noted to develop along the posterior aspect of the vitreous base some time after vitrectomy for posterior segment pathology. These breaks were caused by traction from vitreous incarceration in the sclerotomy incision. ${ }^{9}$ However, giant tears were not seen in these circumstances.

Patients 1,3 and 4 had sustained penetrating trauma before undergoing pars plana vitrectomy. The penetrating trauma was associated with retained intraocular foreign bodies in patients 1 and 4 . Aylward and associates ${ }^{10}$ described giant retinal tears in 14 eyes that had sustained penetrating trauma with or without retained intraocular foreign bodies. Of these eyes, 4 were rendered aphakic before the development of the giant retinal tear, 7 had undergone anterior vitrectomy, and the majority had some degree of anterior vitreous incarceration. The latency of the detachment tended to be greater in the penetrating trauma group than in the blunt trauma group, suggesting that the giant retinal tears after penetrating trauma occur at the time of delayed posterior vitreous detachment, associated with basal gel incarceration.

McLeod $^{6}$ described the development of giant retinal tears associated with detachment of residual vitreous cortex in eyes that had previously undergone a central vitrectomy via the pars plana. $\mathrm{He}$ recommended prophylactic cryotherapy across the location of the posterior border of the vitreous base for $30^{\circ}$ on each side of all pars plana entry sites, extended to $360^{\circ}$ in selected cases. The development of giant retinal tears in these four reported cases has obvious implications for perhaps all young patients with incomplete posterior vitreous separation undergoing pars plana vitrectomy. Carefully monitored $360^{\circ}$ cryotherapy applied to the postoral retina may be recommended in high-risk cases.

Key words: Pars plana vitrectomy, Retinal detachment, Retinal tears, Vitrectomy complications.

\section{REFERENCES}

1. Schepens CL. Retinal detachment and allied diseases. Philadelphia: WB Saunders, 1983:520-55.

2. Scott JD. Giant tear of the retina. Trans Ophthalmol Soc UK 1975;95:142-4.

3. Freeman HM. The vitreous in idiopathic giant retina breaks. In: Schepens CL, Neetens A, editors. The vitreous and vitreoretinal interface. Berlin: SpringerVerlag, 1987:229-40.

4. Weidenthal DT, Schepens CL. Peripheral fundus changes associated with ocular contusion. Am J Ophthalmol 1966;62:465-77.

5. Billington BM, Leaver PK, McLeod D. Management of retinal detachment in the Wagner-Stickler syndrome. Trans Ophthalmol Soc UK 1985;104:875-9.

6. McLeod D. Giant retinal tears after central vitrectomy. Br J Ophthalmol 1985;69:96-8.

7. Faulborn J, Conway BP, Machmer R. Surgical complications of pars plana vitreous surgery. Ophthalmology 1978;85:116-25.

8. Oyakawa RT, Schachat AP, Michels RG, Rice TA. Complications of vitreous surgery for diabetic retinopathy. I. Intraoperative complications. Ophthalmology 1983;90:517-21.

9. Kreiger AE. Wound complications in pars plana vitrectomy. Retina 1993;13:335-44.

10. Aylward GW, Cooling RJ, Leaver PK. Traumainduced retinal detachment associated with giant retinal tears. Retina 1993;13:136-41. 